\title{
PRIMEROS RESULTADOS DE UNA INTERVENCIÓN ENFERMERA SOBRE EL ABORDAJE DEL TABAQUISMO EN PERSONAS CON UN TRASTORNO MENTAL GRAVE
}

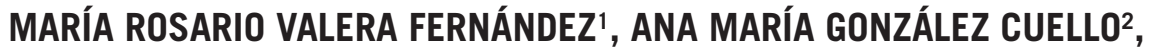 ROSA ACEÑA DOMÍNGUEZ ${ }^{1}$, ANA BELÉN VALERA FERNÁNDEZ' ${ }^{1}$ ANTONIO MORENO POYATO ${ }^{3}$ Y FRANCINA FONSECA CASALS ${ }^{1}$}

${ }^{1}$ Departamento de Adicciones. Instituto de Neuropsiquiatría i Adicciones (INAD).

Consorci Mar Parc de Salut. Barcelona.

${ }^{2}$ Departamento de Enfermería. Escuela Universitaria de Enfermería. Universidad de Murcia.

${ }^{3}$ Departamento de Enfermería. Escuela Superior de Enfermería del Mar, adscrita a la Universitat

Pompeu Fabra. Barcelona.

\section{RESUMEN}

Introducción: se ha encontrado una importante relación entre el consumo de tabaco y distintos trastornos relacionados con la salud mental, observándose una mayor prevalencia de consumo, mayor dependencia a la nicotina, menores intentos de abandono y mayor dificultad para conseguir la abstinencia.

Objetivo: evaluar la efectividad de una intervención enfermera sobre el abordaje del tabaquismo para generar motivación en la reducción del consumo de tabaco o una cesación tabáquica completa a personas con un trastorno mental grave.

Metodología: estudio de tipo analítico cuasiexperimental pre-post, destinado a usuarios de los servicios de hospitalización parcial, completa y atención comunitaria. Se realiza una entrevista inicial, una intervención grupal y seguimiento a los 3,6 y 12 meses.

Resultados: se reduce el número de cigarrillos en 9,4 cigarrillos/día y se aumenta el grado de autoeficacia. Se mantiene el grado de dependencia a la nicotina y la motivación para dejar de fumar. Mejora de los valores de cooximetría y tensión arterial.

Conclusiones: las personas con un trastorno mental están motivadas e interesadas en dejar su consumo de tabaco.

Palabras clave: tabaco, trastorno mental, intervención, enfermería, hospitalización. 


\section{INTRODUCCIÓN}

\author{
«¿Por qué casi todos los jugadores \\ (ludópatas) fuman? [...] \\ ¿Por qué todas las prostitutas y todos \\ los locos fuman?» \\ LEÓN TOLSTól (1828-1910) \\ «Why do men stupefy themselves?» \\ Original en Essay and Letters (1911)
}

Aunque ya hace más de 100 años, Tolstói se preguntaba por qué todos los locos fumaban. Es desde 1986 cuando propiamente la comunidad científica empieza a corroborar una fuerte asociación entre el consumo de tabaco y la presencia de sintomatología psiquiátrica ${ }^{1}$. En estos años, se ha encontrado una importante relación entre el consumo de tabaco y distintos trastornos relacionados con la salud mental, especialmente, depresión, esquizofrenia, trastornos por consumo de sustancias y ansiedad ${ }^{2,3}$.

Son numerosos los estudios que establecen una prevalencia de consumo de tabaco de dos a tres veces superior entre la población con un trastorno mental que en la población general ${ }^{4,5} 0$, según algunas publicaciones recientes, incluso cinco veces superior $^{6}$. Se estima que en los Estados Unidos el $70 \%$ de los cigarrillos consumidos por los estadounidenses son fumados por la población con trastornos mentales ${ }^{7}$.

Además, en la población con trastornos mentales, también se ha descrito ${ }^{8,9}$ :

- Una adicción a la nicotina más elevada, existiendo, además, una relación directamente proporcional entre la intensidad de la clínica psiquiátrica y la gravedad de la adicción.

- Un vínculo con el cigarrillo con características propias y dificultades específicas.

- Menor número de intentos de abandono.

Correspondencia: María Rosario Valera Fernández Correo electrónico: charo-valera@hotmail.com
- Mayor dificultad en la obtención de la abstinencia estable.

Las mayores tasas de prevalencia de consumo tabáquico en personas con un trastorno mental se encuentran dentro del ámbito hospitalario. De León y Díaz ${ }^{3}$ llevaron a cabo un metanálisis de 42 estudios, donde encontraron que la prevalencia de consumo tabáquico era del $68 \%$ en usuarios con esquizofrenia que estaban hospitalizados frente al $57 \%$ de los pertenecientes a la atención comunitaria.

Son varias las teorías que tratan de explicar la mayor vulnerabilidad al consumo de tabaco en las personas con trastornos mentales. Al igual que en la población general, no existe un único factor que haga más vulnerables a estas personas ${ }^{10}$; se considera, más bien, una combinación de factores biológicos, psicológicos y sociales.

Sobre la cesación tabáquica en la población con trastorno mental, el estudio de Shroeder y Morris ${ }^{11}$ muestra el porcentaje de personas que ha conseguido la cesación tabáquica según el tipo de enfermedad psiquiátrica que presenta o la ausencia de esta.

Se observa que ninguna enfermedad psiquiátrica es incapacitante para dejar de fumar y que en todas ellas existe un porcentaje de pacientes que ha conseguido la abstinencia mantenida. Sin embargo, se aprecia que en ninguna enfermedad psiquiátrica se llega a los niveles de cesación de la población sin trastornos, encontrándose grandes diferencias en el caso del trastorno bipolar (del 16\% frente al 42,5\% de la población no psiquiátrica) y en las personas con trastornos psicóticos (del 27,2\% frente al 42,5\%). Los resultados del estudio de Veiga ${ }^{10}$ muestran un fenómeno similar al anterior estudio, observándose cómo las tasas de abstinencia de las personas con un trastorno mental son inferiores a las de la población general.

El objetivo principal del estudio es evaluar la efectividad de una intervención enfermera especializada sobre el abordaje del tabaquismo, para conseguir generar motivación en la reducción del consumo de tabaco o una cesación tabáquica completa a perso- 
nas con un trastorno mental grave que están vinculadas a diferentes servicios de la Red de Salud Mental del distrito de Sant Martí en Barcelona (hospitalización completa, hospitalización parcial y atención comunitaria).

\section{METODOLOGÍA}

El estudio sigue una metodología científica que se basa en una investigación cuantitativa de tipo analítico cuasiexperimental pre-post.

La intervención enfermera del estudio se ha destinado a usuarios vinculados a los servicios de rehabilitación psiquiátrica de media estancia y alta dependencia psiquiátrica como unidades de hospitalización completa; el hospital de día como servicio de hospitalización parcial y los centros de salud como servicios de atención comunitaria, de la Red de Salud Mental del Institut de Neuropsiquiatria i Addiccions (INAD) del Consorci Mar Parc de Salut de Barcelona. Este estudio obtuvo la aprobación del Comité de Ética del Parc de Salut Mar (2016/6855/l).

Los criterios de inclusión en el estudio eran ser usuarios mayores de edad, vinculados a cualquiera de los servicios mencionados anteriormente, que fueran fumadores y que presentaran estabilidad psicopatológica, entendida como la ausencia de cambio de tratamiento durante el último mes. La elección de los usuarios se realizó de forma voluntaria.

El tamaño muestral se calculó de acuerdo con las diferencias esperadas antes-después respecto a la puntuación obtenida en el test de motivación para dejar de fumar de Richmond. Para una prueba de la $t$ de Student de datos relacionados, con una potencia estadística del $80 \%$ y un error alfa de 0,05, se necesitan 24 pacientes para detectar como estadísticamente significativa una diferencia equivalente a dos tercios de desviación estándar. Se asumió una pérdida máxima de seguimiento del $25 \%$.

La intervención sobre el abordaje del tabaquismo propiamente dicha consta de tres partes diferenciales:
1. Entrevista individual. En esta, se realiza la recogida de datos iniciales mediante una anamnesis completa sobre los datos sociodemográficos, los datos clínicos del diagnóstico psiquiátrico, así como los datos relacionados con el consumo de tabaco como: la dependencia a la nicotina mediante el Test de Fagerström; la dependencia psicológica, social y gestual del tabaco a través de la Escala de Glover-Nilsson; la motivación para dejar de fumar con el Test de Richmond; la autoeficacia del usuario ante el abandono tabáquico; el estadio de cambio a través de la Escala URICA; el craving (ansia) de tabaco mediante el Questionnaire on Smoking Urges-Brief (QSU); el síndrome de abstinencia con la escala MNWS (Minnesota Nicotine Withdrawal Scale); así como el control de las medidas biológicas (tensión arterial y CO espirado).

2. Intervención grupal. Se emplea una metodología presencial y una visión participativa, dinámica y motivacional. Se realiza una intervención grupal en cada servicio del estudio. Cada grupo está compuesto de 10 sesiones semanales, con una duración de 90 minutos aproximadamente cada una de ellas (60 minutos para la intervención psicoeducativa, y 30 minutos adicionales para el control de las medidas biológicas). La intervención es de tipo cíclica, en la cual se pueda incorporar un participante en una sesión avanzada sin necesidad de seguir un orden establecido. El material informativo de las sesiones fue de elaboración propia, al no encontrar en la literatura científica material confeccionado para esta población de estudio en concreto. Este material se elaboró con la información de guías validadas, como, por ejemplo: el Programa de Información sobre Tabaquismo de la Región de Murcia del año 2014; la guía para dejar de fumar del Ministerio de Sanidad, Servicios Sociales e lgualdad y el Comité Nacional para la Prevención del Tabaquismo de 2005²; la Guia Práctica Recintes sense Fum de la Xarxa Catalana d'Hospitals sense Fum del 2011³; la Guía de actuación en pacientes fumadores ingresados en 
unidades de salud mental de la Xarxa Catalana d'Hospitals sense Fum de $2012^{14}$, y la guía de práctica clínica Treating Tobacco Use and Dependence: 2008 Update $^{15}$.

La temática trabajada en cada sesión fue:

a. Conocimientos previos. Mitos sobre el tabaco.

b. Conocimientos básicos sobre tabaquismo. Autorregistros.

c. Tipos de cigarrillos. Calendario.

d. Consecuencias del tabaquismo y beneficios de su abandono.

e. Factores motivacionales de mantenimiento y abandono.

f. Síndrome de abstinencia.

g. Tratamiento para la reducción o la cesación completa.

h. Caída y recaída. Utilización de TIC en el tabaquismo.

i. Cigarrillo electrónico. Role-playing.

j. Conocimientos posteriores. Diploma.

3. Seguimiento. Una vez finalizada la intervención grupal, se realiza un seguimiento de los participantes, bien de forma presencial, bien de forma telefónica, a los 3, 6 y 12 meses.

Para el análisis estadístico inicial, se describieron las variables cuantitativas mediante la media aritmética y su correspondiente desviación estándar. Las variables categóricas se describieron mediante tabla de frecuencias (número y porcentaje). Se evaluaron posibles asociaciones entre variables categóricas mediante la prueba de la $\chi^{2}$ de Pearson (o prueba exacta de Fisher en función de la adecuación de la prueba). Se evaluaron posibles diferencias entre períodos de estudio (al inicio y después de la intervención) con el test de los rangos con signo de Wilcoxon (muestras pareadas) para las variables continuas. Se consideraron asociaciones estadísticamente significativas aquellas cuyo nivel de significación estadística $(p)$ era $<0,05$. Los análisis estadísticos se realizaron mediante el paquete estadístico Stata 15.0 software (Stata Corp.; College Station, Texas, Estados Unidos).

\section{RESULTADOS}

Los datos aportados en este estudio son los relativos a aquellos recogidos antes de la intervención y justo después de haberla acabado.

El tamaño muestral es de 25 participantes; de los cuales, 10 (40\%) están vinculados al servicio de hospitalización completa; 6 (24\%), a hospitalización parcial; y los 9 restantes (36\%), a atención comunitaria. De estos, 14 son hombres (56\%) y 9 son mujeres (44\%), con una media de edad de 47,5 años (comprendida entre los 19 y los 62 años).

Sobre los datos clínicos de diagnósticos, encontramos que 12 participantes (48\%) presentan esquizofrenia; 4 (16\%), un trastorno esquizoafectivo; otros 4 (16\%), un trastorno depresivo; 3 (12\%), un trastorno afectivo bipolar; y los 2 participantes restantes (8\%), otros diagnósticos. En este caso, se objetiva una diferencia en la distribución de los diagnósticos en función del servicio al que están vinculados, mostrando una mayor proporción de los trastornos psicóticos en los servicios de hospitalización completa y atención comunitaria, frente a la mayor proporción de trastornos afectivos en la hospitalización parcial.

Las características de consumo de los participantes son un consumo medio de 27,6 cigarrillos/día durante una media de 30,16 años. Han intentado dejar de fumar en 1,72 ocasiones, objetivándose el doble de intentos de abandono entre los participantes de atención comunitaria frente a los servicios de hospitalización parcial y completa. De los participantes, 17 (68\%) han presentado un período de abstinencia anterior, de los cuales, el $80 \%$ refieren no haber presentado de forma subjetiva un empeoramiento de la sintomatología psiquiátrica de su trastorno de base durante este período de abstinencia. El grado de dependencia psicológica es elevado (de 11,92; siendo 0-5: leve; 6-10: moderada; y 11-16: alta), la dependencia social es moderada (de 4,16; resultando 0-2: leve; 3-5: moderada; y 6-8: alta) y el grado de dependencia gestual es leve (de 11,16; siendo 0-15: leve; 16-31: moderada; y >32: alta). Los 
resultados del cuestionario QSU muestran que el nivel de craving $(37,48)$ es elevado, sobre todo, en los participantes de los servicios de hospitalización completa y atención comunitaria. Los síntomas de abstinencia valorados mediante la escala MNWS presentan una puntuación similar en los tres servicios, objetivándose una ligera diferencia no estadísticamente significativa en el servicio de hospitalización parcial (tabla 1).
A partir de los datos que son valorados antes de la intervención y una vez finalizada esta, se encuentra que, respecto al número de cigarrillos consumidos de forma diaria, hay una disminución de 9,4 cigarriIlos menos, resultando estadísticamente significativa (27,6 frente a 18,16; $p<0,001)$. El Test de Fagerström, que evalúa la dependencia a la nicotina, ha descendido en 0,920 puntos, aunque este resultado no es significativo $(6,28$ frente a 5,$36 ; p=0,085)$, al

Tabla 1. Comparación de resultados relacionados con el consumo entre los diferentes servicios

\begin{tabular}{|c|c|c|c|c|}
\hline Variables & $\begin{array}{l}\text { Rehabilitación } \\
\text { en media y larga } \\
\text { estancia }(n=10)\end{array}$ & $\begin{array}{l}\text { Hospitalización } \\
\text { parcial } \\
(n=6)\end{array}$ & $\begin{array}{l}\text { Atención } \\
\text { comunitaria } \\
(n=9)\end{array}$ & $p$ \\
\hline N. ${ }^{\circ}$ de cigarrillos/día & $19,3(13,3)$ & $22(9,90)$ & $40,5(5,83)$ & $\begin{array}{c}0,0008^{*(2)} \\
0,0099 * * *(2)\end{array}$ \\
\hline Craving (QSU) & $39,3(20,14)$ & $27,67(10,89)$ & $42(17,04)$ & $0,3604^{(1)}$ \\
\hline Abstinencia (MNWS) & $11,7(9,22)$ & $9,33(11,34)$ & $11,56(8,26)$ & $0,6434^{(1)}$ \\
\hline $\begin{array}{l}\text { Dependencias } \\
\text { Física (Fagerström) } \\
\text { Psicológica } \\
\text { (Glover-Nilsson) } \\
\text { Social (Glover-Nilsson) } \\
\text { Gestual (Glover-Nilsson) }\end{array}$ & $\begin{array}{l}4,1(2,46) \\
11,7(4,62) \\
4,1(1,72) \\
9,8(3,48)\end{array}$ & $\begin{array}{c}6,83(1,72) \\
11,16(3,92) \\
3,16(2,31) \\
9,5(7,23)\end{array}$ & $\begin{array}{c}8,33(1,00) \\
12,6(3,16) \\
4,8(1,53) \\
13,7(6,28)\end{array}$ & 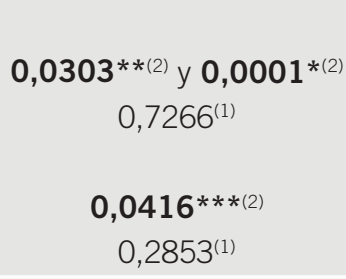 \\
\hline Motivación & $3(2,74)$ & $5,5(2,88)$ & $6,67(2,96)$ & $0,0071^{*(2)}$ \\
\hline Autoeficacia & $2,7(2,54)$ & $4,17(3,31)$ & $5,67(2,65)$ & $0,0145^{*(2)}$ \\
\hline $\begin{array}{l}\text { Estadios de cambio } \\
\text { Precontemplación } \\
\text { Contemplación } \\
\text { Acción }\end{array}$ & $\begin{array}{l}4 \\
6 \\
0\end{array}$ & $\begin{array}{l}0 \\
6 \\
0\end{array}$ & $\begin{array}{l}0 \\
7 \\
2\end{array}$ & $0,049 *(3)$ \\
\hline $\begin{array}{l}\text { Cafeína } \\
\text { N. }{ }^{\circ} \text { de consumidores } \\
\text { Dosis media en mg }\end{array}$ & $\begin{array}{c}8 \\
157,5\end{array}$ & $\begin{array}{c}3 \\
70,83\end{array}$ & $\begin{array}{c}9 \\
179,44\end{array}$ & $\begin{array}{l}0,044 * * *(3) \\
0,024 * * *(1)\end{array}$ \\
\hline
\end{tabular}

*Estadísticamente significativo entre los servicios de rehabilitación de media y larga estancia y atención comunitaria.

**Estadísticamente significativo entre los servicios de rehabilitación de media y larga estancia y hospitalización parcial.

***Estadísticamente significativo entre los servicios de hospitalización parcial y atención comunitaria.

${ }^{(1)}$ Test de rangos de Kruskal-Wallis.

(2) Test de Dunnett.

(3) Prueba exacta de Fisher.

MNWS: Minnesota Nicotine Withdrawal Scale; n: tamaño de la muestra; p: nivel de significación estadística; QSU: Questionnaire on Smoking Urges-Brief. 
igual que pasa con la motivación para dejar de fumar (4,92 frente a 5,60; $p=0,202)$. Respecto al grado de autoeficacia y al nivel de conocimientos sobre el tabaquismo, también resulta estadísticamente significativa la diferencia encontrada entre antes y después de la intervención (tabla 2).

Si se analizan los datos relativos a las medidas biológicas controladas, se obtienen diferencias estadísticamente significativas en cuanto a la cooximetría ( $p=0,005)$, siendo esta de 2,28 en el inicio y de 1,68 tras la intervención (0: entre 0 y 6 p.p.m.; 1: entre 7 y 10 p.p.m.; 2: entre 11 y 15 p.p.m.; 3: entre 16 y 25 p.p.m.; 4: más de 26 p.p.m.). La tensión arterial ha disminuido una media de $2,15 \mathrm{~mm} \mathrm{Hg}$, lo que no es estadísticamente significativo ( $p=0,080$ ). Se ha objetivado que las cifras de tensión arterial sistólica que estaban dentro de la normalidad (no superando los $140 \mathrm{~mm} \mathrm{Hg}$ ) se han mantenido estables, sin embargo, las cifras arteriales sistólicas por encima de la máxima se han visto reducidas.

\section{DISCUSIÓN}

Al igual que en otros estudios recientes, los resultados obtenidos muestran que algunas de las creencias existentes entre las personas con un trastorno mental y su consumo de tabaco no son ciertas. En este sentido, son varias las que se encuentran en la literatura. Por ejemplo, existe la creencia de que las personas con un trastorno mental no tienen motivación e interés en dejar de fumar. Sin embargo, la motivación encontrada en este estudio es de un grado moderado. Igualmente, se cree que el hecho de dejar de fumar empeora la evolución de la enfermedad mental y/o adictiva de base en el paciente y que puede comportar problemas en el ajuste farmacológico del tratamiento antipsicótico ${ }^{16}$. No obstante, el $80 \%$ de los participantes que han presentado un período de abstinencia refieren no haber sufrido un empeoramiento de la sintomatología psiquiátrica del trastorno de base, y el $20 \%$ restante comenta mayores niveles de ansiedad (síntoma propio de la abstinencia a la nicotina). Otra creencia encontrada es que el personal suele temer que las prohibiciones totales de fumar aumenten el estrés y la ansiedad en el paciente, provocando un mayor número de alteraciones de conducta y solicitud de altas voluntarias. Sin embargo, son numerosos los estudios que avalan la aceptación y la eficacia de la prohibición total de fumar en las unidades de hospitalización de salud

Tabla 2. Diferencias de las variables antes y después de la intervención $(n=25)$

\begin{tabular}{|c|c|c|c|c|c|c|c|}
\hline \multirow[b]{2}{*}{ Variable } & \multicolumn{2}{|c|}{ Preintervención } & \multicolumn{2}{|c|}{ Posintervención } & \multicolumn{2}{|c|}{ Diferencia } & \multirow[b]{2}{*}{$p$} \\
\hline & M & DE & M & DE & M & DE & \\
\hline N. ${ }^{\circ}$ de cigarrillos/día & 27,6 & 14,09 & 18,16 & 12,09 & $-9,40$ & 10,81 & 0,0001 \\
\hline Fagerström & 6,28 & 2,62 & 5,36 & 2,72 & $-0,92$ & 2,56 & 0,1094 \\
\hline Richmond & 4,92 & 3,20 & 5,60 & 2,40 & 0,68 & 2,59 & 0,2632 \\
\hline Autoeficacia & 4,12 & 2,96 & 5,32 & 2,23 & 1,20 & 2,08 & 0,0490 \\
\hline Nivel de conocimientos & 6,68 & 1,14 & 8,88 & 1,39 & 2,20 & 1,25 & 0,0001 \\
\hline
\end{tabular}

DE: desviación estándar; M: media; n: tamaño de la muestra; $p$ : nivel de significación estadística.

Las cifras en negrita corresponden a resultados estadísticamente significativos.

Test de rangos de Wilcoxon para muestras pareadas. 
mental, sin haber aumentado las alteraciones conductuales ni el estrés de los pacientes ${ }^{17,18}$. En este estudio, se muestra como los niveles de craving de los participantes son menores en los servicios de hospitalización, donde existe una prohibición total de fumar, objetivándose mayores tasas de craving entre la población de atención comunitaria y una puntuación de sintomatología de la abstinencia similar en los tres servicios. Se puede deducir de este hecho que la prohibición completa de fumar durante la hospitalización sirve de ayuda para el control del consumo de tabaco. En muchas ocasiones, se ha detectado que el personal no suele preguntar sobre el consumo de tabaco a los pacientes, no ofrece consejo sobre el abandono ni propone el tratamiento de forma regular en el caso de que el paciente manifieste interés. Según el estudio de Kulkarni et al. ${ }^{19}$, el $48 \%$ de los profesionales de la salud mental consideran que el tratamiento del tabaquismo en sus pacientes no es su responsabilidad.

En este estudio, no se ha presentado la cesación completa del consumo de tabaco entre los participantes, aunque sí que se ha objetivado un descenso importante del número de cigarrillos diarios. La reducción del consumo de tabaco como paso previo a la cesación completa es una de las estrategias recomendadas por los expertos ${ }^{20}$. Es importante que se valide bioquímicamente con una reducción significativa de los niveles de $\mathrm{CO}$ en el aire espirado, tal y como se ha mostrado en este estudio, con una reducción de 0,6 puntos. El problema principal de la reducción del consumo de cigarrillos es que ninguna cantidad de cigarrillos fumados ha demostrado ser segura. Por eso, se considera que hay una reducción real de daños si la disminución del consumo es un paso que facilite la cesación posterior.

Como limitación principal del estudio, se encuentran las propias de los estudios cuasiexperimentales, como no disponer de un grupo de control o la no aleatorización de la muestra, de ahí que se haya intentado suplir esta limitación con el abordaje en diferentes servicios de salud mental.
Para la realización de este estudio, se ha contado con la Ayuda a Proyectos de Investigación del Colegio Oficial de Enfermería de la Región de Murcia en la convocatoria para el año 2016 y una beca PERIS en la convocatoria 2016-2020.

Los autores declaran que no existe conflicto de intereses en la realización y publicación de este estudio.

\section{CONCLUSIONES}

De los resultados obtenidos en el presente estudio, así como de los encontrados en la literatura científica, se puede deducir que las personas con un trastorno mental están motivadas e interesadas en dejar su consumo de tabaco, no objetivándose un empeoramiento de su trastorno de base durante el período de abstinencia. La prohibición completa de fumar durante la hospitalización supone una oportunidad de abordar esta temática y proporcionar tratamiento para conseguir la cesación. Las intervenciones destinadas a este fin resultan eficaces, como se ha demostrado en este estudio.

\section{BIBLIOGRAFÍA}

1. Bushe C, Haddad P, Peveler R, Pendlebury J. The role of lifestyle interventions and weight management in schizophrenia. J Psychopharmacol. 2005;19(6 Suppl):28-35.

2. Sobradiel N, García-Vicent V. Consumo de tabaco y patología psiquiátrica. Trastor Adict. 2007;9(1):31-8.

3. De León J, Díaz FJ. A meta-analysis of worldwide studies demonstrates an association between schizophrenia and tobacco smoking behaviors. Schizophr Res. 2005;76(23):135-57.

4. Marqués de Oliveira R, Furegato AR. Esquizofrenia y dependencia del tabaco: una revisión integradora. Enferm Glob. 2012;11(25):381-403.

5. Tsoi DT, Porwal M, Webster AC. Interventions for smoking cessation and reduction in individuals with schizophrenia. Cochrane Database Syst Rev. 2013;(2):CD007253.

6. Zvolensky MJ, Farris SG, Leventhal AM, Ditre JW, Schmidt NB. Emotional disorders and smoking: relations to quit attempts and cessation strategies among treatmentseeking smokers. Addict Behav. 2015;40:126-31. 
7. Beck AK, Baker AL, Todd J. Smoking in schizophrenia: cognitive impact of nicotine and relationship to smoking motivators. Schizophr Res Cogn. 2015;2(1):26-32.

8. Eriksen M, Mackay J, Schluger NW, Islami F, Drope J; World Lung Foundation. The tobacco atlas: fifth edition. Atlanta: American Cancer Society; 2015.

9. Minichino A, Bersani FS, Calò WK, Spagnoli F, Francesconi M, Vicinanza R, et al. Smoking behaviour and mental health disorders--mutual influences and implications for therapy. Int J Environ Res Public Health. 2013;10(10): 4790-811.

10. Veiga Rodeiro S. Influencia de la presencia de patología psiquiátrica en la cesación tabáquica: estudio de los pacientes tratados. Santander: Universidad de Cantabria; 2016.

11. Schroeder SA, Morris CD. Confronting a neglected epidemic: tobacco cessation for persons with mental illnesses and substance abuse problems. Annu Rev Public Health. 2010;31:297-314.

12. Guía para dejar de fumar. Madrid: Ministerio de Sanidad, Servicios Sociales e Igualdad y Comité Nacional para la Prevención del Tabaquismo; 2005.

13. Martínez C, Carabasa E (coords.). Guía pràctica Recintes sense fum. Barcelona: Xarxa Catalana d'Hospitals sense Fum, Institut Català d'Oncologia, Departament de Salut de la Generalitat de Catalunya; 2011. Disponible en: https://www.xchsf.cat/docs/125-Guia_Recintes_7. pdf

14. Ballbè M, Cano M, Contel M, Feria I, Hernández R, Pagerols J, et al. Guía de actuación en pacientes fumadores ingresados en unidades de salud mental. Barce-
Iona: Xarxa Catalana d'Hospitals sense Fum, Institut Català d'Oncologia, Departament de Salut de la Generalitat de Catalunya; 2013. Disponible en: https://www. xchsf.cat/docs/461-Guia\%20actuacion.pdf

15. Fiore MC, Jaén CR, Baker TB, Bailey WC, Benowitz NL, Curry SJ, et al.; Tobacco Use and Dependence Guideline Panel. Treating Tobacco Use and Dependence: 2008 Update. Clinical Practice Guideline. Rockville: US Department of Health and Human Services; 2008.

16. Ratschen E, Britton J, McNeill A. Implementation of smoke-free policies in mental health in-patient settings in England. Br J Psychiatry. 2009;194(6):547-51.

17. El-Guebaly N, Cathcart J, Currie S, Brown D, Gloster S. Public health and therapeutic aspects of smoking bans in mental health and addiction settings. Psychiatr Serv. 2002;53(12):1617-22.

18. Harris GT, Parle D, Gagné J. Effects of a tobacco ban on long-term psychiatric patients. J Behav Heal Serv Res. 2007;34(1):43-55.

19. Kulkarni M, Huddlestone L, Taylor A, Sayal K, Ratschen E. A cross-sectional survey of mental health clinicians' knowledge, attitudes, and practice relating to tobacco dependence among young people with mental disorders. BMC Health Serv Res. 2014;14:618.

20. Anthenelli RM, Benowitz NL, West R, St Aubin L, McRae T, Lawrence D, et al. Neuropsychiatric safety and efficacy of varenicline, bupropion, and nicotine patch in smokers with and without psychiatric disorders (EAGLES): a double-blind, randomised, placebo-controlled clinical trial. Lancet (London, England). 2016; 387(10037):2507-20. 